\title{
Conjunctival extranodal marginal zone lymphoma of mucosa-associated lymphoid tissue in the fornix: do not overlook conjunctival lymphomas
}

This article was published in the following Dove Press journal:

Clinical Ophthalmology

29 March 2013

Number of times this article has been viewed

\author{
Masayuki Hata' \\ Kazuaki Miyamoto' \\ Ken Ogino' \\ Shinji Sumiyoshi \\ Nagahisa Yoshimura' \\ 'Department of Ophthalmology \\ and Visual Sciences, ${ }^{2}$ Department \\ of Diagnostic Pathology, Kyoto \\ University Graduate School of \\ Medicine, Sakyo, Kyoto, Japan
}

Correspondence: Masayuki Hata Department of Ophthalmology and Visual Sciences, Kyoto University Graduate School of Medicine, Sakyo-ku, Kyoto 606-8507, Japan

Tel $+8|7575| 3250$

Fax +8I 757520933

Email trj74h6@kuhp.kyoto-u.ac.jp
Background: Here we report three cases of conjunctival lymphoma that were initially unnotified or misdiagnosed as other ocular diseases because of the small tumor size, peripheral tumor location (the tumor was hidden in the fornix), and nonspecific symptoms.

Methods: Three patients diagnosed with conjunctivitis or nasolacrimal duct obstruction were referred to our clinic because they were unresponsive to standard medical treatments. Routine anterior segment examination did not reveal any lesions, but further careful examination with a strong eyelid draw revealed minimally elevated tumors in the peripheral fornix under the lid. Excisional biopsies were performed.

Results: Histopathologic and immunohistologic examinations indicated the presence of extranodal marginal zone lymphoma of mucosa-associated lymphoid tissue (MALT lymphoma). All patients underwent additional radiation therapy. There was no evidence of recurrence in any patient during the follow-up period.

Conclusion: Detection of conjunctival lymphoma can be challenging. If no apparent lesion is present and the patient has nonspecific symptoms, the inner surface of the eyelid should be carefully examined. Elaborate eyelid eversion, with eyeball movement, should be performed to avoid misdiagnosing or overlooking peripheral conjunctival lymphomas.

Keywords: MALT lymphoma, conjunctival lymphoma, misdiagnosis, fornix

\section{Introduction}

Extranodal marginal zone lymphoma of mucosa-associated lymphoid tissue (MALT lymphoma) is the most common lymphoid neoplasm of the conjunctiva. It is characterized by painless, salmon-pink patches in the fornix or bulbar conjunctiva, and has an indolent clinical course. ${ }^{1-3}$ Conjunctival lymphoma is relatively easy to diagnose when it presents as an apparent mass with the characteristic salmon-pink appearance. However, it is sometimes challenging to detect and/or recognize when it presents in a patient who has nonspecific symptoms, including irritation, epiphora, and mass sensation. Adding to the difficulty, conjunctival lymphoma often presents as an obscure lesion that mimics the appearance of other ocular surface diseases, such as allergic or chronic conjunctivitis. Increased awareness and prompt identification of these tumors are necessary to avoid oversight and misdiagnosis, and the resultant delays in beginning treatment and possible systemic involvement. In this report, we document three nearly identical cases of MALT lymphoma that were localized to the conjunctival fornices. No patient was immunocompromised or had other disease. In all three cases, the tumors were initially unnotified and patients were misdiagnosed because of nonspecific symptoms and also because of the peripheral location and small tumor size. 


\section{Case report I}

A 62-year-old woman was referred from a private clinic with the diagnosis of refractory conjunctivitis. She complained of irritation and hyperemia in her right eye that had lasted for weeks. She did not respond to topical medical therapy (antibiotics, glucocorticoids, and nonsteroidal anti-inflammatory drugs). No lesion was found during slit-lamp biomicroscopy with a simple eyelid draw in the right eye. However, an elaborate eyelid draw, with eyeball movement to the opposite side, revealed a small, red-colored mass that infiltrated the bulbar in the lower and upper peripheral fornices (Figure 1A and B). No other systemic or orbital lesions were found, even on contrast-enhanced computed tomography images of the orbit. The diagnosis of MALT lymphoma was confirmed by biopsy. Immunohistologic examination showed diffuse $\mathrm{B}$ cell proliferation and kappa light chain restriction (Figure 2A-C). Following radiation therapy (30 Gy in 15 fractions), no evidence of lesion recurrence was seen in a one-year follow-up period.

\section{Case report 2}

A 33-year-old woman with a 4-month history of epiphoria was referred to our clinic with a diagnosis of nasolacrimal duct obstruction. Because of nonresponsiveness to topical medical therapy, further examinations were performed and revealed multiple lesions hidden under the lid (Figure 1C

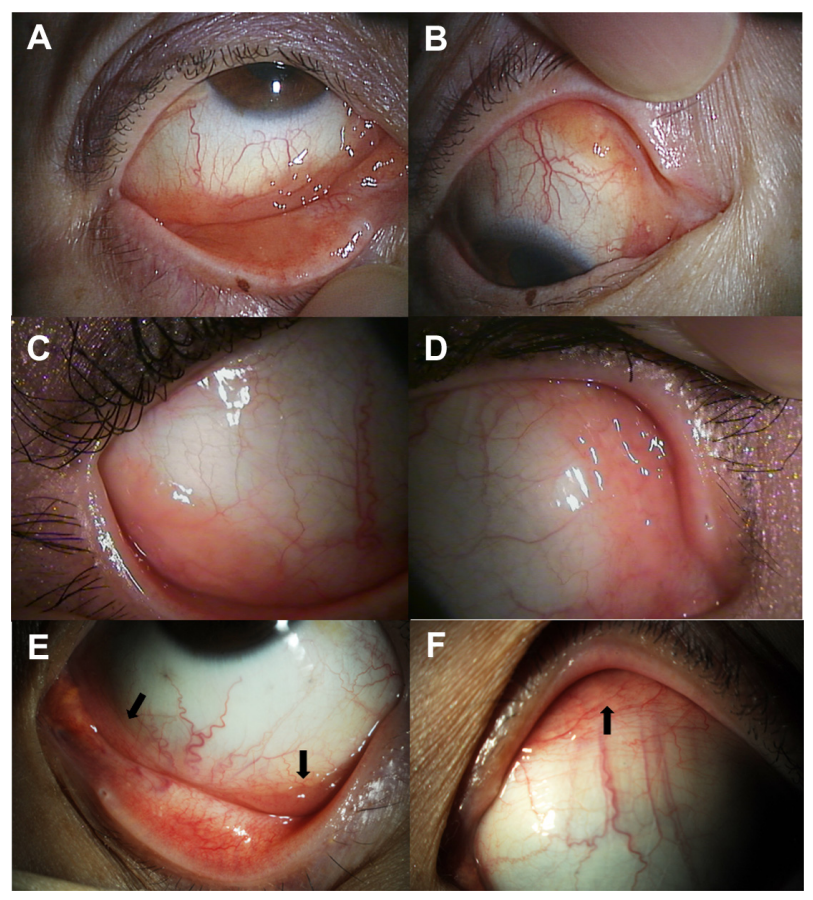

Figure I Slit-lamp examination with strong eyelid eversion revealed a conjunctival tumor located in the fornix in case I (A and B), case 2 (C and D), and case 3 (E and F). and D). Biopsy of the conjunctival masses was performed and the pathology report was consistent with a diagnosis of MALT lymphoma (Figure 2D). There was no evidence of systemic involvement or residual malignant tissue. The patient was successfully treated with radiation therapy (30 Gy in 15 fractions). No evidence of lesion recurrence was seen in a 6-month follow-up period.

\section{Case report 3}

A 40-year-old woman with a 6-month history of intermittent redness in the left eye was referred to our clinic. She was initially diagnosed with chronic conjunctivitis, but did not respond to topical medical therapies. Further examination showed multiple lesions hidden in the peripheral fornix under the lid (Figure 1E and F). Biopsy of the conjunctival mass was performed, and the histologic findings were consistent with those of MALT lymphoma (Figure 2E). There was no evidence of systemic involvement or residual malignant tissue. The patient was successfully treated with radiation therapy (30 Gy in 15 fractions). There was no evidence of lesion recurrence in a 6-month follow-up period.

\section{Discussion}

Here, we describe three patients with MALT lymphoma who presented with minimally elevated tumors hidden in the peripheral fornix. They were initially misdiagnosed with chronic conjunctivitis or nasolacrimal duct obstruction, and the lymphoma was unnotified. Because they did not respond to standard medical treatment (antibiotics, glucocorticoids, and nonsteroidal anti-inflammatory drugs), more thorough examinations were performed and trivial lesions were detected. Finally, histopathologic examination of biopsied tissue allowed the correct diagnosis to be made.

Ocular involvement in malignant lymphoma can include orbital, conjunctival, eyelid, uveal, and vitreal localization. Most conjunctival lymphomas are MALT lymphomas, according to the Revised European American Lymphoma classification and the new World Health Organization classification. It is sometimes difficult to differentiate between pseudolymphoma and MALT lymphoma in small samples using histology and immunohistochemistry. The patients have a relatively good prognosis, and radiation therapy is generally effective in treating primary ocular MALT lymphomas. ${ }^{4,5}$ Unfortunately, systemic lymphoma can develop and tumors may recur in the ipsilateral or contralateral eye. ${ }^{6,7}$ It has been previously reported that the rate of development of systemic lymphoma was $38 \%$ at 5 years and $79 \%$ at 10 years. ${ }^{6}$ In addition, because many patients are asymptomatic, it can take many months or even years to detect tumor recurrence. Patients with MALT 


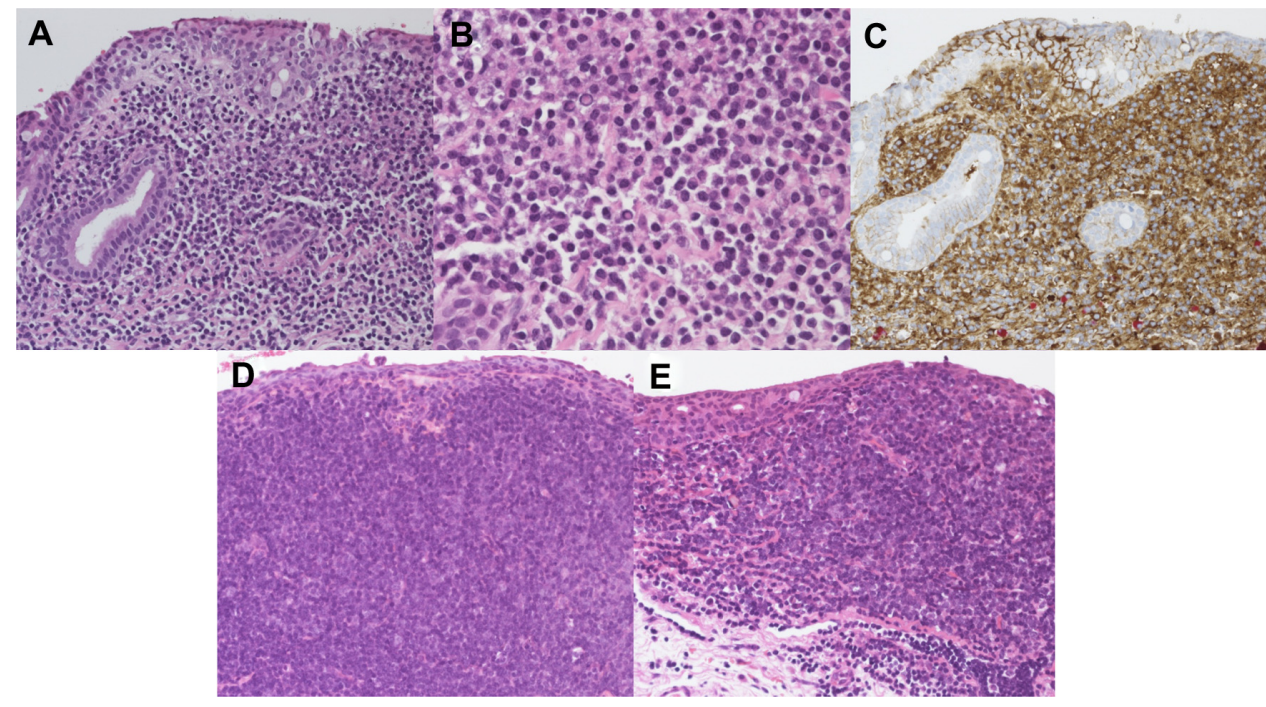

Figure 2 Histologic examination stained with hematoxylin and eosin demonstrated a diffuse proliferation of lymphoid cells in the conjunctiva in case I (A and B), case 2 (D), and case 3 (E). Immunohistochemical double stain revealed kappa light chain restriction in case I (C, brown for kappa chain and red for lambda chain). (A, C, D, E 20× and B 40x).

lymphomas have a relatively favorable outcome, but require frequent follow-up indefinitely.

In the three cases presented here, tumors were localized to the peripheral fornices of the conjunctiva and hidden in the eyelid. They appeared as multiple, isolated, minimally elevated lesions. Despite the presence of multiple lesions, routine conjunctival examination failed to detect the lesions. Further careful examination using strong eversion of the eyelid, with eyeball movement, allowed visualization of the abnormal lesions. Shields et $\mathrm{al}^{6}$ reported that about half of conjunctival lymphomas arise in the fornix, with lesions mainly involving the superior or inferior fornices, as in the present cases. Careful examination, including the inferior and superior fornices, should be performed, so as not to overlook trivial lesions in the early stage of development.

There have been reports of conjunctival lymphoma mimicking allergic or chronic conjunctivitis. ${ }^{8-10}$ In these reports, patients presented with atypical, normal-colored, papilla-like lesions or inflammation in both eyes. In this case series, two patients were initially diagnosed with conjunctivitis and one patient with nasolacrimal duct obstruction. All cases were nonresponsive to conventional medical therapies. When patients do not respond to treatment as expected, the patient should be carefully re-examined using strong eyelid eversion with eyeball movement. On the basis of our experience with these three patients, there is a possibility that some conjunctival MALT lymphomas are missed or misdiagnosed.

\section{Disclosure}

The authors report no financial conflicts of interests in this work.

\section{References}

1. Coupland SE, Hummel M, Stein H. Ocular adnexal lymphomas: five case presentations and a review of the literature. Surv Ophthalmol. 2002;47(5):470-490.

2. Johnson TE, Tse DT, Byrne GE Jr, et al. Ocular-adnexal lymphoid tumors: a clinicopathologic and molecular genetic study of 77 patients. Ophthal Plast Reconstr Surg. 1999;15(3):171-179.

3. Stefanovic A, Lossos IS. Extranodal marginal zone lymphoma of the ocular adnexa. Blood. 2009;114(3):501-510.

4. Hardman-Lea S, Kerr-Muir M, Wotherspoon AC, Green WT, Morell A, Isaacson PG. Mucosal-associated lymphoid tissue lymphoma of the conjunctiva. Arch Ophthalmol. 1994;112(9):1207-1212.

5. Suzuki J, Ohguro H, Oguri N, et al. Clinicopathologic and immunogenetic analysis of mucosa-associated lymphoid tissue lymphomas arising in conjunctiva. Jpn J Ophthalmol. 1999;43(3):155-161.

6. Shields CL, Shields JA, Carvalho C, Rundle P, Smith AF. Conjunctival lymphoid tumors: clinical analysis of 117 cases and relationship to systemic lymphoma. Ophthalmology. 2001;108(5):979-984.

7. Matsuo T, Ichimura K, Yoshino T. Spontaneous regression of bilateral conjunctival extranodal marginal zone B-cell lymphoma of mucosaassociated lymphoid tissue. J Clin Exp Hematop. 2007;47(2):79-81.

8. Lee DH, Sohn HW, Park SH, Kang YK. Bilateral conjunctival mucosa-associated lymphoid tissue lymphoma misdiagnosed as allergic conjunctivitis. Cornea. 2001;20(4):427-429.

9. Akpek EK, Polcharoen W, Ferry JA, Foster CS. Conjunctival lymphoma masquerading as chronic conjunctivitis. Ophthalmology. 1999; 106(4):757-760.

10. Akpek EK, Polcharoen W, Chan R, Foster CS. Ocular surface neoplasia masquerading as chronic blepharoconjunctivitis. Cornea. 1999;18(3):282-288. 


\section{Publish your work in this journal}

Clinical Ophthalmology is an international, peer-reviewed journal covering all subspecialties within ophthalmology. Key topics include: Optometry; Visual science; Pharmacology and drug therapy in eye diseases; Basic Sciences; Primary and Secondary eye care; Patient Safety and Quality of Care Improvements. This journal is indexed on
PubMed Central and CAS, and is the official journal of The Society of Clinical Ophthalmology (SCO). The manuscript management system is completely online and includes a very quick and fair peer-review system, which is all easy to use. Visit http://www.dovepress.com/ testimonials.php to read real quotes from published authors. 